\title{
Forschungsstelle für Jagdkultur an der Universität Bamberg
}

Unter Leitung von Frau Dr. Sigrid SCHwenk trat zu Ende des Jahres 1980 in Bamberg die Forschungsstelle für Jagdkultur ins Leben. Zu ihrem Entstehen und ihren Aufgaben wird der nachfolgende Text wiedergegeben.

$\mathrm{Da}$ die naturwissenschaftlich orientierten Gebiete der Jagdwissenschaft wie die Wildbiologie bereits von mehreren deutschen Universitäten betreut werden, sollen in Bamberg in klarer Abgrenzung hierzu die mit der Jagd zusammenhängenden geisteswissenschaftlichen und kulturellen Probleme im Vordergrund stehen, um eine seit langem empfundene Lücke zu schließen. Zu den Aufgaben werden einerseits die Ermittlung von Fakten zu aktuellen Problemen, andererseits Untersuchungen im Bereich der jagdwissenschaftlichen Grundlagenforschung gehören.

Nach Herausgabe des großen historisch-philologischen Wörterbuchs der deutschen Jägersprache soll die damit verbundene umfangreiche Materialsammlung fortgeführt und ein gekürztes Handwörterbuch

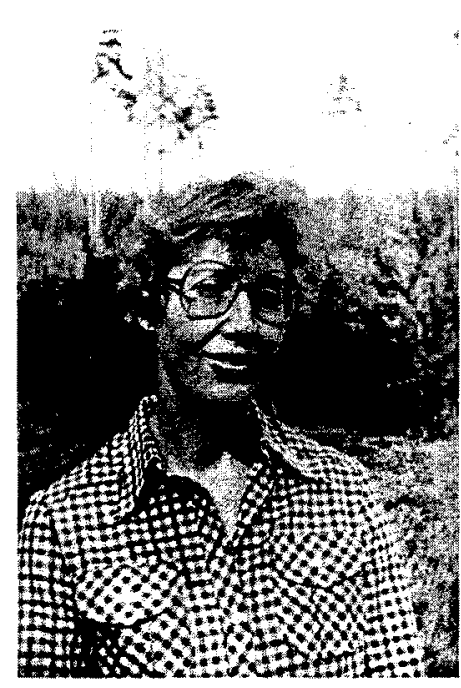

Dr. SIGRID SCHWENK für den praktischen Gebrauch des deutschen Jägers erarbeiter werden. Darüber hinaus wird die erste größere Leistung die Fertigstellung einer zuverlässigen Bibliographie der gesamten deutschsprachigen Jagdliteratur für die Zeit von 1851 bis 1980 sein, deren Fehlen von der jagdwissenschaftlichen Forschung bislang besonders schmerzlich empfunden wurde. Ein weiteres Anliegen ist die laufende Erfassung aller Neuerscheinungen auf dem Gebiet des jagdlichen Schrifttums und die periodische Herausgabe einer Titelsammlung. Vorgesehen und bereits eingeleitet sind ferner Untersuchungen statistischer Natur, vor allem um den Wildbiologen und Okologen Wildstandsveränderungen im geschichtlichen Ablauf durch Ermittlung zuverlässiger Daten deutlich zu machen. Diese Ergebnisse werden zugleich Unterlagen für die Beurteilung der wirtschaftlichen Bedeutung der Jagd in Vergangenheit und Gegenwart liefern. Brauchtumsgepflogenheiten sollen im Einvernehmen mit der Jägerschaft überprüft und gegebenenfalls auf historischer Basis abgesichert werden. Das gleiche gilt für Untersuchungen zur Geschichte des deutschen Jagdrechts, das wegen seiner großen Differenzierung häufig genug zu Mißverständnissen und Fehlinterpretationen in der Offentlichkeit geführt hat.

Frau Dr. SCHWenk steht bei ihrer Forschungsarbeit ein Beirat zur Seite, zu dem Prof. Dr. R. Bergmann, Bamberg, der Präsident des Landesjagdverbandes Bayern, Dr. G. Frank, München, Ministerialrat Dr. P. Leonhardt, München, und Prof. Dr. Dr. h. c. K. LINDNER, Bamberg, gehören.

$\mathrm{Zu}$ der Ubernahme der Forschungsstelle gratulieren wir Frau Dr. SCHWENk herzlich und wünschen ihr Arbeitserfolge wie bisher.

E. UECKERMANN

\section{Herausgabe der Zeitschrift Cynegeticus}

In den Vereinigten Staaten hat sich eine Gruppe von Hochschullehrern verschiedener Disziplinen zusammengeschlossen, die angesichts der weltweiten Erfolge des ausschließlich naturwissenschaftlich ausgerichteten wildlife management sicherstellen möchte, daß auch die geisteswissenschaftliche Seite des komplexen Phänomens Jagd angemessen Pflege und Beachtung erfährt. Die von diesem Kreis begründete Zeitschrift führt den Titel 